\title{
Modelización simulada en tiempo real de la evolución de un incendio mediante el Método Fast Marching
}

\author{
Adrián Bargueño Juárez \\ Expace on Board Systems, abarguenojuarez@gmail.com \\ Dr. Luis Santiago Garrido \\ Universidad Carlos III, sgarrido@ing.uc3m.es \\ Dr. Luis Moreno \\ Universidad Carlos III, moreno@ing.uc3m.es
}

\begin{abstract}
Resumen
Conseguir un modelo que pueda predecir la evolución de un incendio ha sido, y aún sigue siendo, un importante campo de investigación. Durante muchos años modelos físico-químicos, empíricos y mixtos han ido perfeccionándose y acercándose cada vez más a la evolución real de los incendios, a pesar de la complejidad de los factores implicados en el comportamiento del fuego. Pero desde hace unas décadas, se ha venido desarrollando modelos computacionales que permiten predecir la evolución de un incendio. El estudio que se presenta en este artículo, intenta modelizar la propagación de un incendio utilizando el método Fast Marching, el cual asemeja la evolución del incendio a la propagación de una onda, mediante la resolución de la ecuación Eikonal. Los primeros resultados que se han conseguido permiten presentar el modelo de forma visual mediante una Interfaz Gráfica de Usuario, en la cual se facilita la introducción de valores de variables como el viento y el tiempo, y en la que la modelización del incendio se presenta mediante gráficos que permiten su interpretación de forma intuitiva.
\end{abstract}

Palabras Clave: Fast Marching, Fast Marching Cuadrado, ecuación Eikonal, Modelización de un incendio

\section{INTRODUCCIÓN}

Los incendios forestales constituyen una de las principales amenazas de parques naturales, bosques y montes, donde ocasionan graves pérdidas ambientales, económicas y personales. Por ello disponer de modelos fiables que predigan el comportamiento del fuego permite incrementar la seguridad y la eficacia de la extinción de los incendios.
Se han elaborado diferentes modelos desde distintos puntos de vista y utilizando también medios diferentes. Modelos físicos, basados en las leyes universales de la física y de la química, Dupuy y Larini [2], modelos empíricos, basados en el análisis estadístico de datos obtenidos en fuegos experimentales realizados en laboratorio o en campo, McArthur [6], y modelos mixtos, también denominados semi-empíricos donde se conjuga el empirismo y la teoría.

En las últimas décadas se han elaborado también modelos de computador para conformar la propagación de un incendio. El enfoque más común ha sido simular la propagación del fuego como un proceso discreto de encendidos a través de una rejilla regularmente espaciada, denominados modelos celulares. El modelo Kourtz y O’Regan [4], calcula el tiempo que tarda el fuego en viajar entre ocho celdas vecinas o nodos en una rejilla rectangular. En general, los modelos celulares han tenido un éxito cada vez menor en la reproducción de las formas bidimensionales esperadas y los patrones de crecimiento, ya que las condiciones ambientales se vuelven más heterogéneas.

El enfoque de este estudio está siendo abordar el problema de la modelización de la propagación de un incendio aplicando el método Fast Marching Cuadrado $\left(\mathrm{FM}^{2}\right)$, que consiste en aplicar el método Fast Marching (FMM) dos veces. El método Fast Marching utiliza la métrica L2 en lugar de métrica L1 de métodos similares como Dijkstra [1], y hace una interpolación intrínseca que nos permite resolver de manera eficiente la ecuación continua de propagación de la luz, ecuación Eikonal, para calcular el camino más corto en tiempo en el cual se propagará el incendio.

En otras palabras, aunque el método Fast Marching utiliza datos obtenidos a partir de una rejilla discreta, resuelve la propagación de la luz de forma continua y 
por tanto la solución es también una función continúa.

Por lo tanto este modelo permitirá simular en tiempo real cómo va a ser la propagación del fuego, considerando el frente de propagación como la propagación de una onda, dando el tiempo de llegada del frente del fuego en cada punto del mapa, considerando como entradas el campo vectorial del viento en cada punto, así como la vegetación y la orografía del terreno, especialmente el gradiente.

\section{MATERIALES Y MÉTODOS}

\subsection{METODO FAST MARCHING CUADRADO $\left(\right.$ FM $\left.^{2}\right)$}

El método Fast Marching Cuadrado, desarrollado por L.S. Garrido y L. Moreno [5], es un método muy utilizado en diversos problemas de planificación de movimientos, como planificación de movimientos de robots, aprendizaje de movimientos, generación de hojas de rutas, etc. Este método consiste en aplicar dos veces el Método Fast Marching propuesto por J.A. Sethian [3]. El objetivo de FMM es generar mapas de distancias aproximados, dicho en otras palabras, dado un punto en el espacio, FMM computa la distancia desde ese punto al resto de los puntos en el espacio.

El Método Fast Marching puede ser entendido considerando la expansión de una onda. Por ejemplo si una piedra es lanzada en un estanque, un frente de onda es originado, y esa onda se expande con una forma circular alrededor del punto donde la piedra cayó. En este caso al ser el fluido siempre agua la velocidad de expansión de la onda es siempre la misma y por eso tiene una forma circular, sin embargo si el medio de expansión de la onda es no homogéneo el frente de onda se expande a diferentes velocidades en cada medio. FMM considera que la velocidad de propagación no es la misma en todos los puntos, pero si considera la velocidad en todos los puntos como no negativa, por lo que la onda siempre avanza hacia delante.

Suponiendo que el estanque estuviese formado por dos líquidos de diferentes densidades, como por ejemplo agua y aceite, el tiempo de llegada del frente de onda a cada punto del estanque será diferente debido a esa diferencia de propagación de la onda, por lo que FMM a través de la ecuación Eikonal, asigna un tiempo de llegada a cada punto del estanque. La propagación del frente de onda siempre es hacia los puntos con menor tiempo de llegada, tal y como se muestra en la figura 1, donde el punto negro es el punto donde cayó la piedra y los puntos grises son los puntos donde se computa el FMM.
Como se puede observar la onda se expande hacia los puntos colindantes con el punto negro en función de su tiempo de llegada.

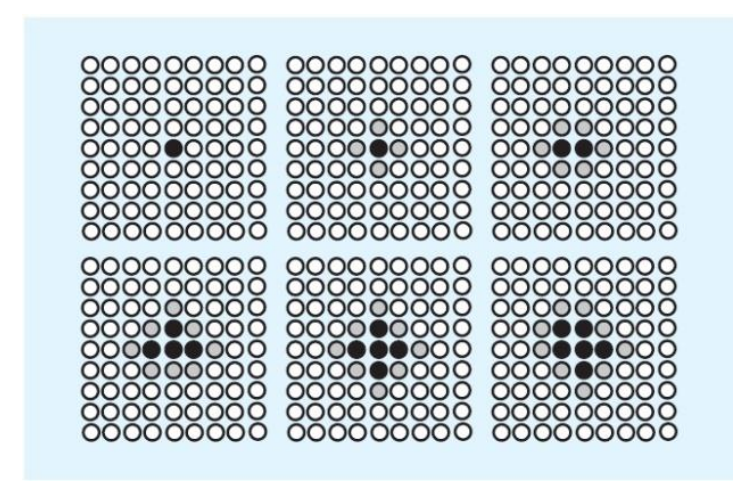

Figura 1: Iteración de la expansión de la onda

Resumiendo, FMM provee una rápida solución aproximada mediante la simulación de la propagación de una onda a través de un ambiente no homogéneo, en el cual la velocidad de propagación depende de la actual posición del frente de onda.

Para ejecutar el método $\mathrm{FM}^{2}$ el primer paso es computar el mapa de velocidades. A cada punto del espacio es dado una velocidad relativa directamente proporcional a la distancia de dicho punto al obstáculo más cercano, es decir, cuanto menor se la distancia de un punto del espacio a un obstáculo menor será la velocidad asociada a ese punto en el mapa de velocidades.

El segundo paso es aplicar FMM desde el punto final y expandir la onda hasta que alcance la actual posición del punto que se considere como inicial. En este caso el mapa de distancias creado se interpreta como un mapa de tiempo de llegada, en el cual cada punto tiene asignado un valor que representa el tiempo que le lleva a la onda alcanzar dicho punto desde el punto fuente, mientras es restringido por el mapa de velocidades.

El paso final es obtener el camino, mediante la aplicación del gradiente descendiente del mapa de distancias, desde el punto inicial hasta que el punto final es alcanzado. Además, el mapa de tiempo de llegadas no tiene mínimo local, por lo que el punto final siempre se alcanza.

La figura 2 ilustra los diferentes pasos del algoritmo FM . 


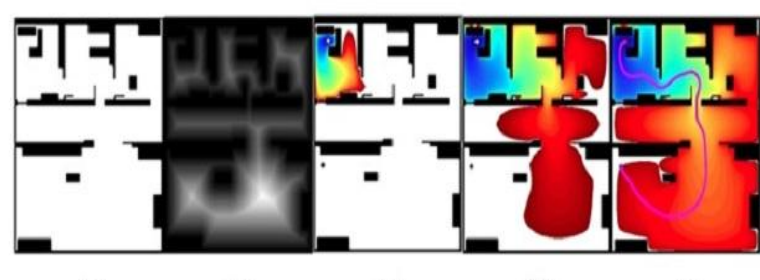

$\begin{array}{lllll}\text { (a) } & \text { (b) } & \text { (c) } & \text { (d) } & \text { (e) }\end{array}$

Figura 2: Pasos del algoritmo FM . a) Mapa binario inicial. b) Mapa de velocidad generado con FMM.

c),d) Propagación de la onda desde el punto final.

e) Camino final mostrado sobre el mapa de tiempo de llegada

\subsection{LA ECUACIÓN EIKONAL Y EL MÉTODO FAST MARCHING}

Una forma de caracterizar la posición de un frente de expansión es computar el tiempo de llegada $\mathrm{T}$, en el cual el frente alcanza cada punto del subyacente espacio matemático de la interfaz.

Para una dimensión podemos obtener la ecuación de la función del tiempo de llegada $\mathrm{T}$, considerando el hecho de que la distancia $\theta$ es el producto de la velocidad $\mathrm{F}$ por el tiempo $\mathrm{T}$

$$
\theta=\mathrm{F} * \mathrm{~T}
$$

La derivada espacial de la solución se convierte en el gradiente

$$
1=\mathrm{F} * \frac{\mathrm{dT}}{\mathrm{d} \theta}
$$

Y por lo tanto la magnitud del gradiente de la función del tiempo de llegada $T(\theta)$ es inversamente proporcional a la velocidad

$$
\frac{1}{\mathrm{~F}}=|\nabla \mathrm{T}|
$$

Para múltiples dimensiones el mismo concepto es válido, porque el gradiente es ortogonal a los conjuntos de nivel de la función de llegada $T(\theta)$. En este sentido podemos caracterizar el movimiento del frente como una solución de un problema de soluciones de contorno. Si la velocidad F depende solo de la posición, entonces la ecuación 3 puede ser reformulada como la ecuación Eikonal

$$
|\nabla \mathrm{T}| * \mathrm{~F}=1
$$

El método de Fast Marching es un algoritmo numérico para resolver la ecuación Eikonal en una malla rectangular ortogonal.

\subsection{IMPLEMENTACIÓN DEL ALGORITMO DE FAST MARCHING EN UNA MALLA ORTOGONAL}

El método Fast Marching se aplica sobre fenómenos que pueden ser descritos como un frente de onda que se propaga de forma normal a sí mismos con una función de velocidad $\mathrm{F}=\mathrm{f}(\mathrm{i}, \mathrm{j})$. La idea principal es construir metódicamente la solución usando solamente valores hacia delante, la llamada condición de entropía. Sea T (i,j) la superficie de la solución T en el que la curva cruza el punto $(i, j)$, entonces satisface la ecuación 4, la ecuación Eikonal.

Con el objetivo de entender cómo funciona el método Fast Marching, imaginemos que un visitante imprudente deja un fuego sin extinguir en algún lugar de una reserva forestal. La llama rápidamente se convierte en un incendio forestal que se expande hacia el exterior. El fuego consume los árboles que va alcanzando, por lo que el fuego siempre se propaga hacia delante. Podemos registrar la posición del frente del fuego en diferentes puntos en el tiempo, y parece que el fuego se expande en la ruta con el menor tiempo de propagación, y por tanto en la longitud más corta si la velocidad es constante.

En acústica y óptica este fenómeno es conocido como el Principio de Fermat o el principio de menor acción. El principio de Fermat establece que la luz viaja entre dos puntos siempre eligiendo el camino más rápido. Por lo que la propagación ocurre de menor a mayores valores de $\mathrm{T}$.

El algoritmo clasifica los puntos de la malla en tres tipos: negros, rojos y verdes. Los puntos negros son puntos donde el tiempo de llegada ya ha sido computado y no va a cambiar en el futuro (son puntos que ya han sido consumidos por las llamas). Los puntos verdes son puntos que no han sido procesados aún, por lo que el tiempo de llegada no ha sido computado todavía (corresponderían a árboles vivos). Los puntos rojos son los puntos pertenecientes el frente de propagación de la onda, considerados como una interfaz entre los puntos negros y los putos verdes de nuestra malla triangular (correspondientes a arboles en llamas en nuestro ejemplo)

Inicialmente solo el punto fuente se marca como negro, y todos los puntos adyacentes a dicho punto fuente se marcan en rojo. En cada iteración el punto rojo con el valor más pequeño de tiempo de llegada $\mathrm{T}(\mathrm{x})$ pasa a formar parte del conjunto de puntos negros. El proceso continuará hasta que todos los puntos se conviertan en puntos negros o el frente de llama alcance el punto final. 
La ecuación Eikonal se aplica a los puntos de la cuadrícula, que pueden ser clasificados, de forma análoga a la calcificación expuesta anteriormente, en tres tipos:

- Puntos vivos, puntos en los que los valores de $\mathrm{T}$ son conocidos

- Puntos de Prueba, puntos alrededor de la curva (puntos vivos) donde la propagación del frente de onda será computado. Este conjunto de puntos son conocidos como banda estrecha. En la siguiente iteración estos puntos pasarán a formar parte del conjunto de los puntos vivos y la banda estrecha avanzará.

- Puntos Lejanos, son puntos donde la propagación no ha sido computada todavía. Según avance las iteraciones y la propagación, estos puntos pasarán formar parte del conjunto de los puntos de prueba.

En la figura 3a) se explica la evolución del método. En la primera subfigura el punto azul oscuro es el punto inicial (fuente) de la onda y los puntos vecinos están marcados en gris. En la segunda subfigura el valor $\mathrm{T}$ de los puntos vecinos ya ha sido computado $\mathrm{y}$ se marcan dichos puntos en azul claro, mientras que los puntos vecinos a estos se etiquetan como puntos grises a la espera de ser computados. La computación continúa hasta que todos los puntos están etiquetados con un color en función del tiempo de llegada.

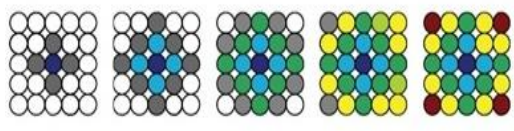

(a) Iterations of FM with one wave in 2D.

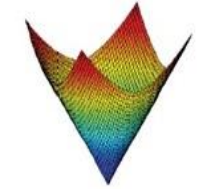

(b) Time of arrival potential $D(\mathbf{x})$ (third axis)
Figura 3: Evolución Método Fast Marching

En la figura 3b) se representa el potencial del tiempo de llegada en 3D

\subsection{Interfaz Gráfica de Usuario (GUI)}

Para permitir introducir los valores de las distintas variables y presentar la modelización de la propagación de un incendio, lo primero que se ha realizado en el proyecto, ha sido crear una GUI (Interfaz Gráfica de Usuario) en Matlab, que permite introducir el valor de las variables directamente y de forma rápida e intuitiva, a partir de datos conocidos, como pueda ser la velocidad del viento medido en distintas estaciones meteorológicas. La interfaz permite ir actualizando el valor de las variables y por tanto conocer en tiempo real el comportamiento del fuego.

En la figura 4 se muestra una visión general de la GUI generada, en la que se muestra cuatro divisiones de la misma. En la parte superior se encuentran dos gráficos, el de la izquierda muestra el camino más corto desde un punto inicial, considerado foco del incendio, y un punto final, que se considera como el punto en el que se quiere conocer cuando será alcanzado por el incendio. En el gráfico superior de la derecha se muestra la forma de propagación del incendio, asemejado a la propagación de una onda, con el tiempo de llegada a cada punto del plano, representado por un color diferente.

En la parte inferior a la izquierda se encuentran las dos variables, viento y tiempo, que se pueden modificar, para actualizar su valor en el caso del viento, o para ver qué zonas serán afectadas por el incendio transcurrido un cierto tiempo.

Por último en la parte inferior a la derecha se muestra las coordenadas en el plano XY del foco del incendio, que se pueden modificar a tiempo real, $\mathrm{y}$ las coordenadas del punto en el que queremos conocer cuando será alcanzado el incendio.

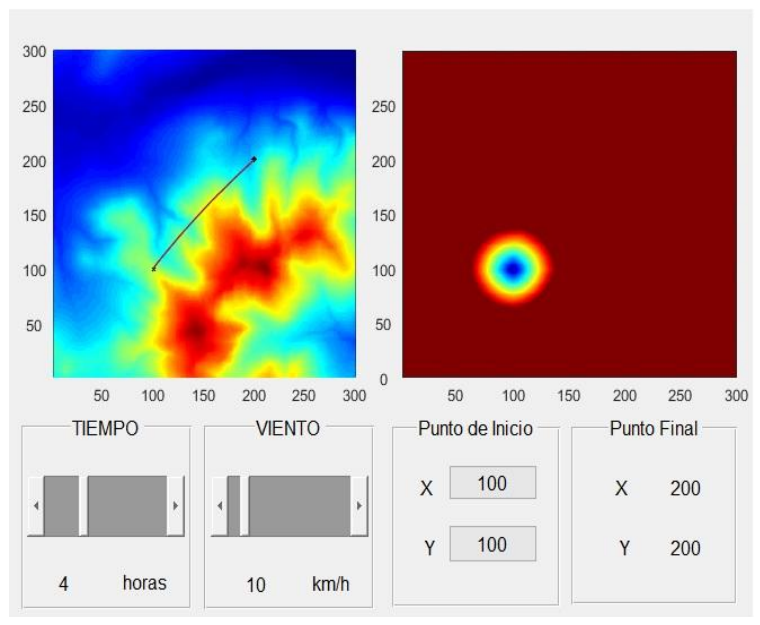

Figura 4: Interfaz Gráfica de Usuario

\section{CONCLUSIONES Y TRABAJO FUTURO}

En este apartado se presentan los primeros pasos que se han dado y los primeros resultados obtenidos en el proyecto, para poder conseguir un modelo que sea capaz de modelar la propagación del fuego en un incendio. El modelo final que se pretende conseguir deberá permitir la entrada de datos puntuales de viento, brisas marinas y vientos de ladera. Asimismo, deberá tener en cuenta el tipo de vegetación en cada 
punto, si es monte bajo, bosque y el tipo de plantas en cada punto del terreno, y deberá tener en cuenta la orografía, la pendiente en cada punto del terreno.

Uno de los primeros objetivos que se ha conseguido en este primer modelo, tal y como se presentan en la figura 5 , ha sido poder situar el foco del incendio en diferentes puntos dentro de una cuadrícula que representa una determinada expansión de terreno.

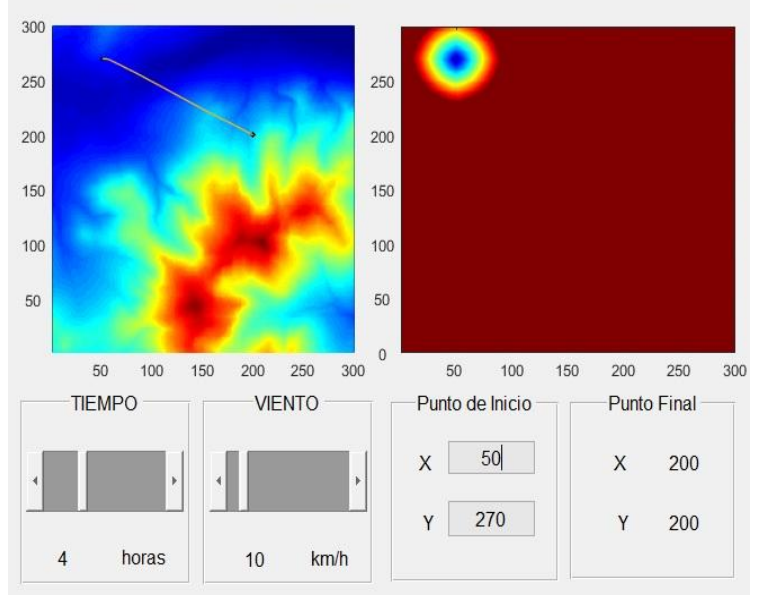

Figura 5: Foco de incendio distinto

Como se puede observar en la gráfica de la izquierda, el método de Fast Marching calcula cuál es el camino más corto en tiempo, en el que fuego se propagaría desde el nuevo punto considerado como foco (punto inicial) hasta el punto en el que se quiere conocer cuando será alcanzado por el frente de propagación (punto final). Este cálculo da la posibilidad de conocer el tiempo que tardaría el frente del fuego en llegar a un punto determinado, como podría ser una población, y poder tomar las medidas necesarias para la evacuación o la preparación de equipos de extinción en dicha localización.

Otros de los avances conseguidos en el proyecto, ha sido la posibilidad de computar la evolución del fuego a lo largo del tiempo.

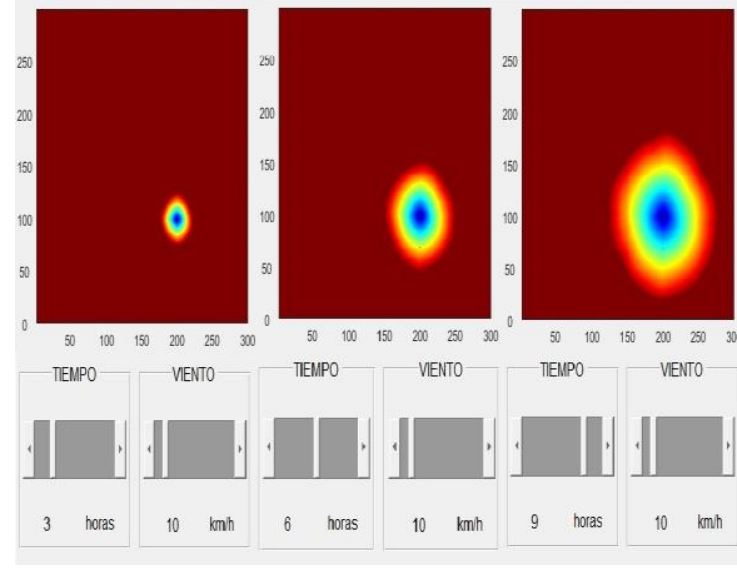

Figura 6: Evolución del incendio con el tiempo

Tal y como se observa en la figura 6 , se ha modelado como sería la evolución del incendio a distintas horas, y con ello se contempla las zonas geográficas que estarían afectadas por el incendio en el trascurso de ese tiempo. Tal y como se muestra en los gráficos se pude tener una aproximación del tiempo que transcurrirá hasta que el incendio alcance una determinada zona, ya que se representa, con distintos colores, el tiempo que tardaría el frente del incendio en alcanzar las zonas colindantes al punto donde comenzó el incendio.

Por último, se ha computado como se vería afectada la propagación del fuego con diferentes valores del viento.

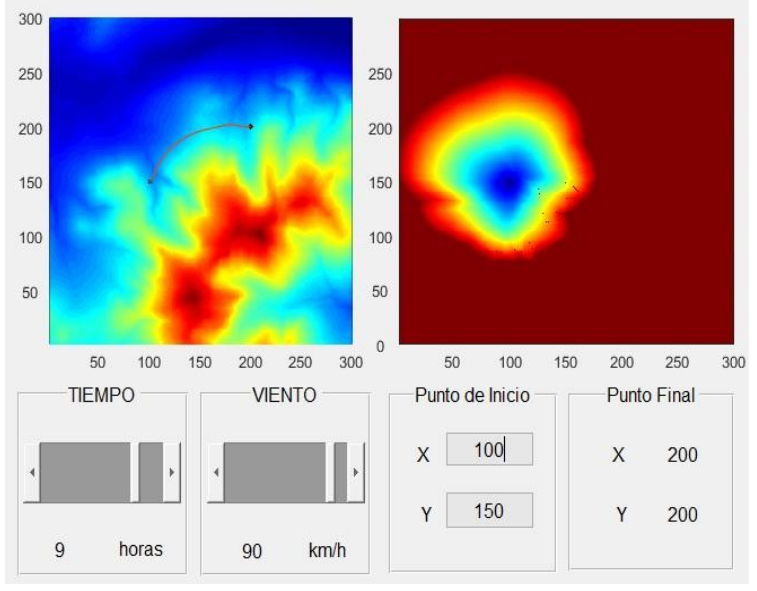

Figura 7: Variación de la propagación del incendio en función de la variable del viento

Como se observa en la figura 7 , la propagación del fuego, en este caso, ya no se produce en circunferencias concéntricas al foco de inicio del incendio, como se observa en la figura 6 , donde la incidencia del viento era pequeña, sino que en este caso se ve afectado por una mayor influencia del viento $\mathrm{y}$ el incendio tiende a propagarse de forma 
más heterogénea. Según se aumente el valor del viento más irregular será la propagación del incendio.

Al modificar el valor de la variable del viento, en el gráfico de la izquierda, se observa que también se ve afectado el camino más corto en tiempo, como se muestra para dos casos diferentes en la figura 8 , desde el foco del incendio hasta el punto final, se produce una curvatura del camino fruto de la acción del viento.

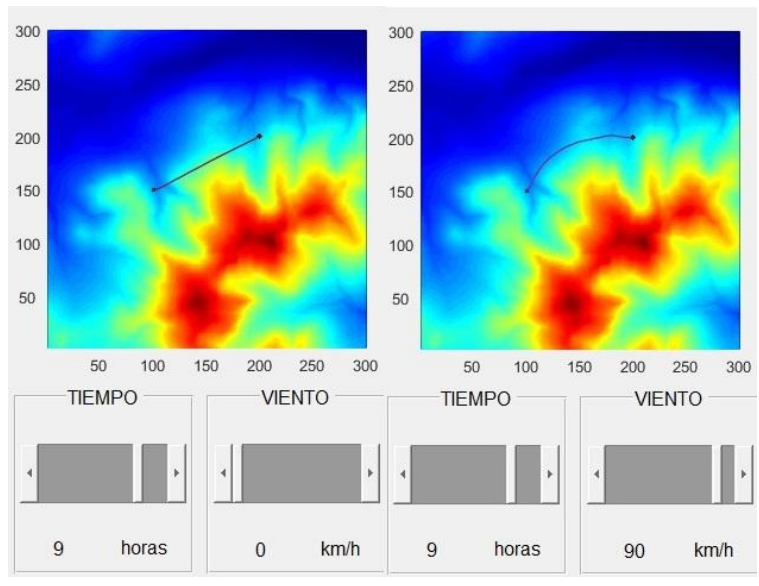

Figura 8: Evolución del camino más corto, en tiempo, en función del viento

Esto también afecta al tiempo de llegada del incendio al punto final, debido a que si el viento es favorable puede hacer que el incendio llegue antes al punto final, pero si es desfavorable el tiempo hasta alcanzar el punto final aumentaría.

Por lo tanto, tal y como se ha explicado, este primer modelo permiten que se puedan ir modificando los valores del viento según se conozcan dichos valores, por ejemplo con los datos recogidos en distintas estaciones meteorológicas cercanas a la localización geográfica donde ha ocurrido el incendio y con ello se consigue modelar la propagación del incendio en tiempo real. Lo que permite poder tomar o varias decisiones en función de la evolución observada en el modelo.

Los siguientes pasos a seguir realizando en el proyecto para alcanzar un modelo final lo suficientemente completo como para presentar una modelización de la propagación de un incendio lo más real posible, serán mejorar la presentación de los datos en la GUI de Matlab, representando la orografía del terreno en 3D y adaptando el método Fast Marching a dicha orografía, pudiendo hacer más intuitiva la comprensión de los datos presentados en la GUI. A su vez se incorporarán más variables al modelo como la propia orografía del terreno, la distinta vegetación y distintas plantas de cada terreno, así como la diferencia entre viento, brisas marinas y vientos de ladera.

\section{Referencias}

[1] Dijkstra Edsger, 1959 Algoritmo de Dijkstra

[2] Dupuy, J-L and Larini M., 1999. Fire spread through porous forest fuel beds : a radiative and convective model including fire-induced flow effects.

[3] J.A. Sethian. (1996). A fast marching level set method for monotonically advancing fronts. Proc. Natl Acad Sci 93:1591-1595

[4] Kourtz, P.H. \& O'Regan, W.G 1971. A model for a small forest fire...to simulate burned and burning areas for use in a detection model. 1971.

[5] L. S. Garrido \& L. Moreno 2015. Motion Planning Using Fast Marching Squared Method

[6] McArthur AG (1966) 'Weather and grassland fire behaviour.'

[7] M. Guijarro, C. Hernando Lara, C. Díez Galilea, J. Madrigal del Olmo 2004. Modelización del comportamiento del fuego en los incendios forestales: Modelos empíricos de predicción de la velocidad de propagación desarrollados en túnel de viento

[8] Robot 2015: Second Iberian Robotics Conference: Advances in Robotics 